\title{
Quantifying the Limits of Detection of Surface-Enhanced Infrared Spectroscopy with Grating Order-Coupled Nanogap Antennas
}

\author{
Aurelian John-Herpin, ${ }^{\circledR}$ Andreas Tittl, ${ }^{\circledR}$ and Hatice Altug*º \\ Institute of Bioengineering, École Polytechnique Fédérale de Lausanne (EPFL), Lausanne 1015, Switzerland
}

Supporting Information

ABSTRACT: Infrared spectroscopy is widely used for biomolecular studies, but struggles when investigating minute quantities of analytes due to the mismatch between vibrational cross sections and IR wavelengths. It is therefore beneficial to enhance absorption signals by confining the infrared light to deeply subwavelength volumes comparable in size to the biomolecules of interest. This can be achieved with surfaceenhanced infrared absorption spectroscopy, for which plasmonic nanorod antennas represent the predominant implementation. However, unifying design guidelines for such systems are still lacking. Here, we introduce an experimentally verified framework

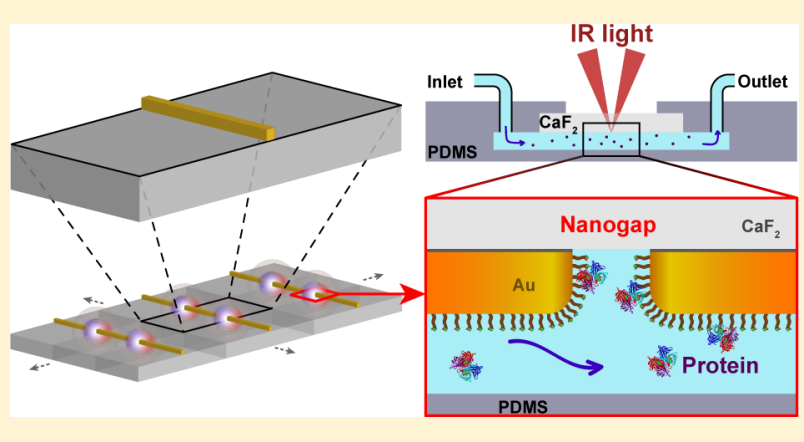
for designing antenna-based molecular IR spectroscopy sensors. Specifically, we find that in order to maximize the sensing performance, it is essential to combine the signal enhancement originating from nanoscale gaps between the antenna elements with the enhancement obtained from coupling to the grating order modes of the unit cell. Using an optimized grating order-coupled nanogap design, our experiments and numerical simulations show a hotspot limit of detection of two proteins per nanogap. Furthermore, we introduce and analyze additional limit of detection parameters, specifically for deposited surface mass, in-solution concentration, and secondary structure determination. These limits of detection provide valuable reference points for performance metrics of surface-enhanced infrared absorption spectroscopy in practical applications, such as the characterization of biological samples in aqueous solution.

KEYWORDS: nanoplasmonics, infrared spectroscopy, protein biosensors

I nfrared (IR) spectroscopy is a powerful analytical technique that provides remarkable insights into the molecular world. IR light can excite molecular vibrations whose resonance frequencies depend on the chemical nature of the bonds as well as their configuration and surroundings. ${ }^{1}$ This technique is particularly useful for investigating the structure and behavior of biomolecules in their native environment, since it operates without the need for extrinsic labels. For instance, in proteins, amide bonds make up the backbone of their structure and can support IR active molecular vibrations such as the amide I and II bands at around 1650 and $1550 \mathrm{~cm}^{-1}$, respectively. ${ }^{2}$ These intrinsic labels can be targeted with IR sensors to provide chemically specific detection. Beyond the mere identification of analytes, the amide I absorbance signature can be analyzed to retrieve information about molecular secondary structure and conformation. ${ }^{3}$ This information, which can be acquired in real-time, can be enhanced with precise three-dimensional structures of biomacromolecules obtained with complementary techniques such as X-ray crystallography and nuclear magnetic resonance (NMR) spectroscopy. ${ }^{4}$

In order to get relevant insights about biomolecular interactions, it is often crucial to probe them in physiological conditions, that is, at low concentrations in aqueous solution or at the submonolayer level. ${ }^{5}$ The requirements of high sensitivity and water-compatibility can usually not be fulfilled by traditional IR spectroscopy techniques, in which large sample concentrations are necessitated and strong vibrational bands of water mask much of the proteins' amide I absorption signature, which holds most of the information related to secondary structure. ${ }^{6}$ Surface-enhanced IR absorptin spectroscopy (SEIRAS) can overcome these limitations by utilizing resonant nanoantennas, which can strongly enhance the light absorption of near-surface analytes. ${ }^{7-11}$ In recent years, the interest in SEIRAS and its application for biological, chemical, and gas sensing has been increasing with the use of plasmonic excitations in metallic and graphene resonators as well as high$Q$ resonant modes of all-dielectric metasurfaces. ${ }^{12-15}$ In metallic IR antennas, the incoming light can be coupled efficiently due to the large oscillator strength of localized surface plasmon resonances (LSPR). In contrast to traditional refractometric LSPR sensors, SEIRAS provides chemical and conformational specificity.

In this work, we introduce a plasmonic grating ordercoupled nanogap (GONG) design to probe the limits of detection for protein sensing and find that we can detect as little as two proteins per nanogap. To provide reference points for performance metrics of SEIRAS in practical applications,

Received: June 24, 2018

Published: September 10, 2018 

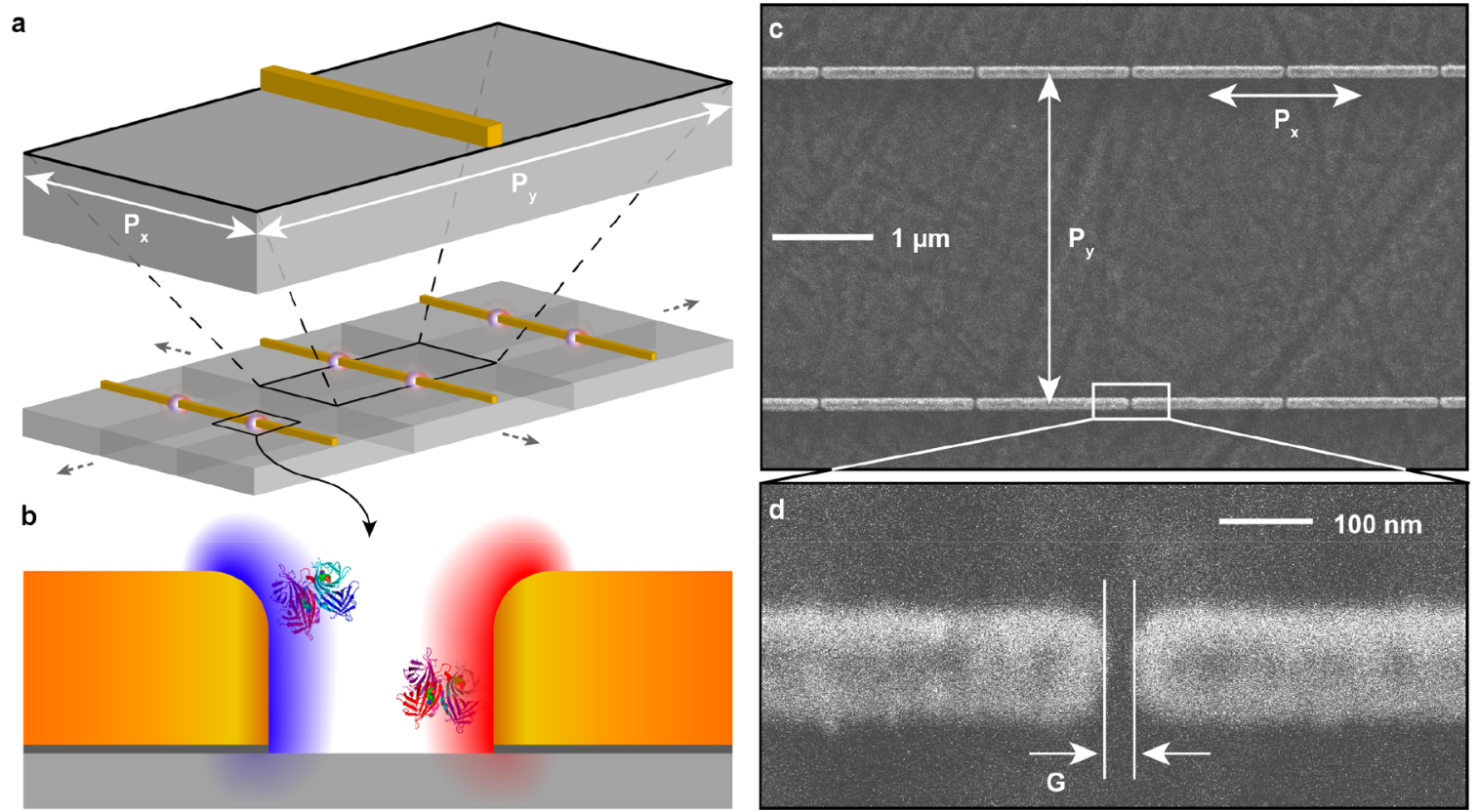

Figure 1. (a) 3D model of the grating order coupled nanogap (GONG) antenna unit cell and array. (b) Schematic representing two streptavidin proteins localized in a nanogap hotspot in which the electromagnetic fields are represented in red and blue gradients (not to scale). (c) Scanning electron microscope (SEM) image displaying the periodicities $P_{x}$ and $P_{y}$ of a GONG array. (d) SEM image of a nanogap with a width of G.

we present additional sensitivity assessment criteria: limits of detection for (i) deposited surface mass, (ii) in-solution concentration, and (iii) secondary structure analysis. We demonstrate the chemically specific detection of proteins in aqueous solution with concentrations as low as $100 \mathrm{pg} \cdot \mathrm{mL}^{-1}$ and resolve their secondary structure content with concentrations down to $500 \mathrm{ng} \cdot \mathrm{mL}^{-1}$. Conventionally, the analysis of protein secondary structure using IR absorbance spectroscopy in aqueous solution is very challenging due to interfering water absorbance and requires orders of magnitude higher concentrations with traditional methods. ${ }^{6}$ The presented quantitative study of proteins using optimized resonant antennas shows that SEIRAS can achieve limits of detection that are highly competitive with other optical label-free biosensors, ${ }^{16}$ with the additional benefits of chemical and conformational specificity.

In our study, gold antennas on an IR-transparent calcium difluoride $\left(\mathrm{CaF}_{2}\right)$ substrate are implemented, since this material system can provide resonances with large excitation cross sections and strong near-field enhancements required for efficient SEIRAS. ${ }^{13,17,18}$ Another advantage of gold is its biocompatibility with well-established surface functionalization protocols. Furthermore, on-chip plasmonic nanoantennas allow for straightforward integration with microfluidics. ${ }^{18-23}$ Regarding resonator shape, nanorods are the most common plasmonic structures for SEIRAS, as they can be tuned to access different spectral ranges and fabricated easily and reproducibly. Their dipolar-like shape makes them inherently efficient at generating high electric near-field enhancements necessary for amplifying molecular vibrations. ${ }^{24}$ Given the important role of these antenna systems in SEIRAS, it is important to maximize their sensing performance.

Nanogaps, that is, spacings between plasmonic structures below a hundredth of the excitation wavelength, can generate strong field enhancements ${ }^{25}$ and are thus interesting for SEIRAS applications. ${ }^{26-31}$ Another effective method for increasing sensitivity is to make use of the enhancement originating from grating order coupling. ${ }^{32,33}$ Traditionally, plasmonic sensing geometries only focus on optimizing one of these aspects, limiting the available design space. We present a comprehensive framework for determining an optimal antenna unit cell geometry that efficiently combines these two enhancement methods simultaneously. Specifically, we demonstrate the importance of independently tuning the unit cell $x$ - and $y$-periodicities to simultaneously achieve analyteaccessible nanogaps and strong grating order coupling. Figure 1a displays a tilted view of a GONG sensor, which consists of unit cells with nonequal periodicities $P_{x}$ and $P_{y}$, each containing a single rod antenna. As a consequence of this geometrical arrangement, the nanogaps arise between the adjacent unit cells along the $x$-direction of the array. Figure $1 \mathrm{~b}$ is a schematic of a nanogap with two streptavidin proteins in the electromagnetic hotspot region (nanogap surface). The dimensions of the array parameters can be visualized in Figure $1 c$,d, which showcase SEM micrographs. The gap has a width $G=32 \mathrm{~nm}$ and the periodicities are $P_{x}=1516 \mathrm{~nm}$ and $P_{y}=$ $3204 \mathrm{~nm}$.

We obtained the optimized GONG design by performing extensive numerical simulations in which we vary both the gap size $G$ as well as the strength of the grating order coupling, which is controlled by the parameter $P_{y}$. Numerical simulations are performed for multiple GONG designs in a parameter space of gap size and $y$-periodicity, where each data point is designated by the pair $\left\{G, P_{y}\right\}$. Specifically, we focus on gap sizes between 20 and $100 \mathrm{~nm}$, as this encompasses the relevant range for protein studies (Figure 2a, bottom). Periodicities are selected so that the associated lowest frequency grating orders are tuned over the resonance frequency of the antennas. We benchmark the performance of these $\left\{G, P_{y}\right\}$-sensor designs by comparing their maximum absorbance for a $5 \mathrm{~nm}$ thick model protein layer placed on the surface of the nanogaps (Figure 2a, top). The protein layer is modeled with custom optical 


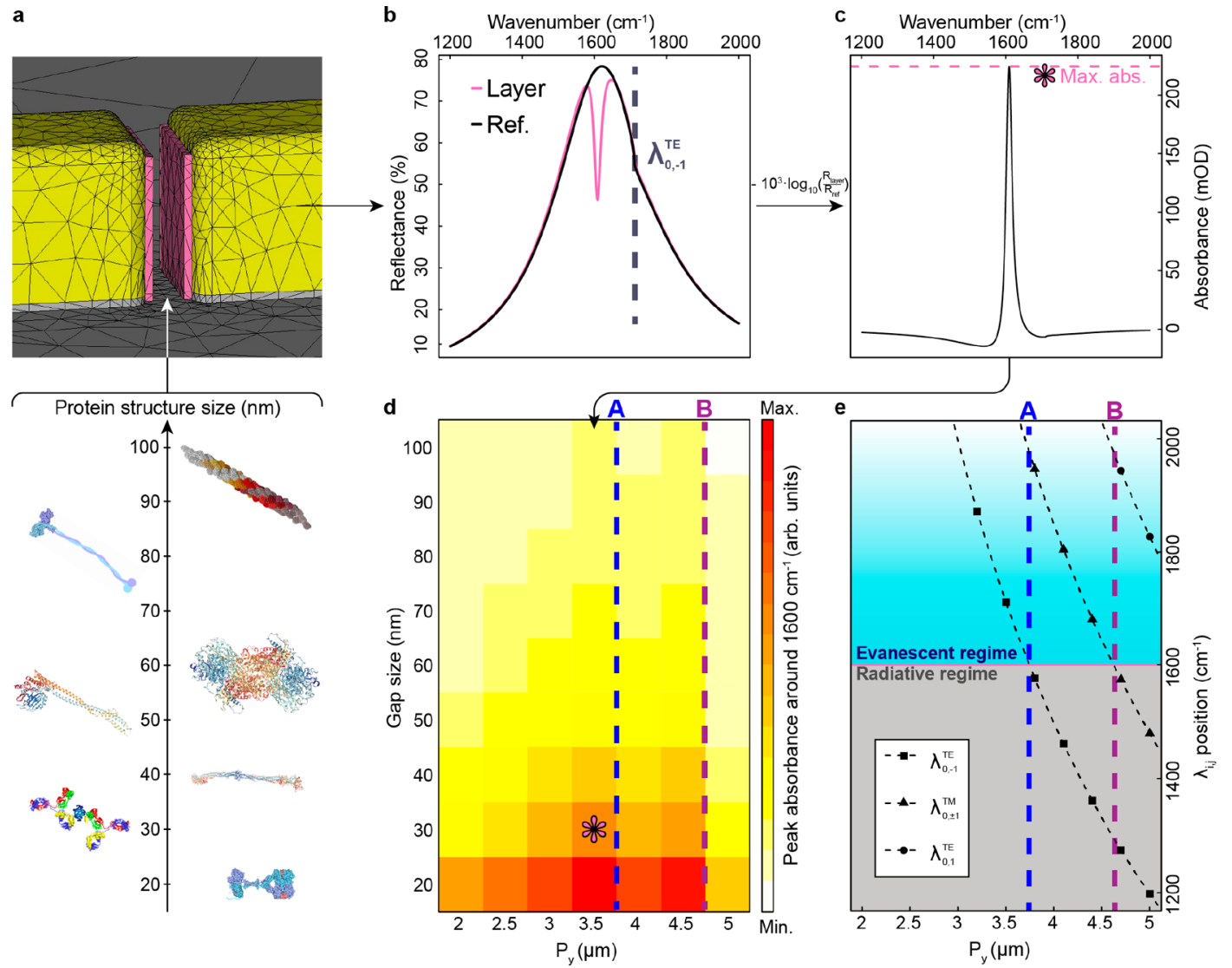

Figure 2. (a) Lower panel: Size of exemplary protein structures on a scale from 20 to $100 \mathrm{~nm}$. From smallest to largest: epidermal growth factor (David Goodsell, doi: 10.2210/rcsb pdb/mom 2010 6), IgG antibodies sandwiching an antigen, fibrinogen (PDB ID: 1M1J, ${ }^{34}$ ), SMC protein (PDB ID: 5XEI, ${ }^{35}$ ), tripeptidyl peptidase II (PDB ID: $3 \mathrm{LXU},{ }^{36}$ ), kinesin (David Goodsell, doi: 10.2210/rcsb_pdb/mom_2005_4), and amyloid protofibril (reproduced in part from Vestergaard et al. ${ }^{37}$ under the Creative Commons Attribution license, CC BY 4.0). Upper panel: Meshed 3D model of a $30 \mathrm{~nm}$ gap with a $5 \mathrm{~nm}$ thick model protein film (pink layer) on the nanogap surfaces as used in the numerical simulations. (b) Reflectance spectra $R_{\text {layer }}$ and $R_{\text {ref }}$ obtained from numerical simulations of a $\left\{G=30 \mathrm{~nm}, P_{y}=3.5 \mu \mathrm{m}\right\}$ sensor with and without model protein layer, respectively. The vertical dashed line at the kink indicates the spectral position of the corresponding grating order. (c) Absorbance spectrum calculated from the reflectance spectra given in panel b. (d) Heatmap displaying comparative absorbance strength as a function of gap size $G$ and $y$ periodicity $P_{y}$. The asterisk indicates the array parameters used for the calculations in panels a-c. (e) Spectral position against $P_{y}$ for the three lowest frequency grating orders (dashed curves). The two vertical dashed lines labeled A and B indicate the critical periodicities for which each of the two lowest frequency grating orders transitions from high to low frequency side of the near-field resonance peak at $1600 \mathrm{~cm}^{-1}$ (pink horizontal line) and thereby causes a transition from evanescent (blue gradient area) to radiative regime (gray shaded area).

constants in order to have its extinction peak at $1600 \mathrm{~cm}^{-1}$, between the amide I and II bands, and with the nondispersive component of the refractive index equal to 1 .

Each $\left\{G, P_{y}\right\}$ sensor is adjusted to provide a near-field resonance maximum at $1600 \mathrm{~cm}^{-1}$, and the structures are then simulated both with and without model protein layer in order to obtain the reflectance spectra (Figure $2 b$ ), from which we calculate the absorbance spectra (Figure 2c). Using the maximum absorbance of each point of the $\left\{G, P_{y}\right\}$ parameter space, we generate a heatmap allowing us to compare the performance of the sensors (Figure 2d).

This map shows that both the gap size and the $y$-periodicity strongly influence the performance of the sensors. Notably, for a given gap size, we observe that performance is highest for designs directly to the left of the dashed lines A and B. This behavior can be understood by considering the position of the lowest frequency grating modes with respect to the localized resonance frequency of the antennas. These grating orders $\lambda_{i, j}$ are commonly accessed at the conditions of $i=0$ and $j= \pm 1$ and can be described by the following equations derived from previous works: ${ }^{38,39}$

$$
\begin{aligned}
& \lambda_{0, \pm 1}^{\mathrm{TM}}=P_{y} \cdot \sqrt{n_{\mathrm{s}}^{2}-\sin ^{2} \theta} \\
& \lambda_{0, \pm 1}^{\mathrm{TE}}=P_{y} \cdot\left(n_{\mathrm{s}} \mp \sin \theta\right)
\end{aligned}
$$

Here, $n_{\mathrm{s}}$ is the refractive index of the antennas' surroundings, $\theta$ is the incident light's inclination angle, TE corresponds to transverse electric, and TM corresponds to transverse magnetic light polarization (see section 1 in the Supporting Information for more details). These equations confirm that only the $y$ periodicity of the GONG unit cell needs to be tuned in order to achieve strong coupling to the grating order at the lowest frequency. Consequently, $P_{x}$ could be freely adjusted to achieve a desired nanogap size $G$ and resonance position, which can be tuned by choosing the right antenna length $L$. This results in the following condition for the $x$-periodicity of the GONG unit cell:

$$
P_{x}=L+G
$$

Using eqs 1 and 2, we can plot the grating order positions as a function of $P_{y}$ (Figure 2e). We observe that the lines A and B 
a

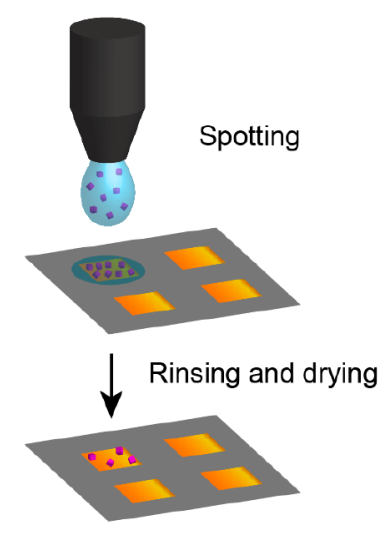

d

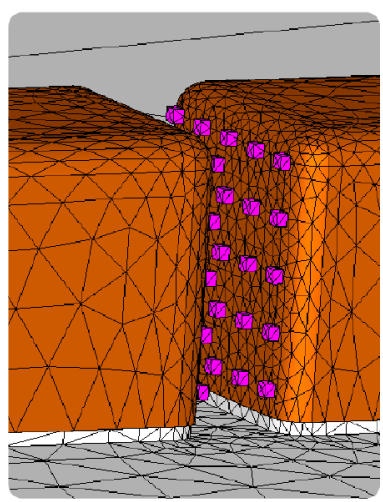

b

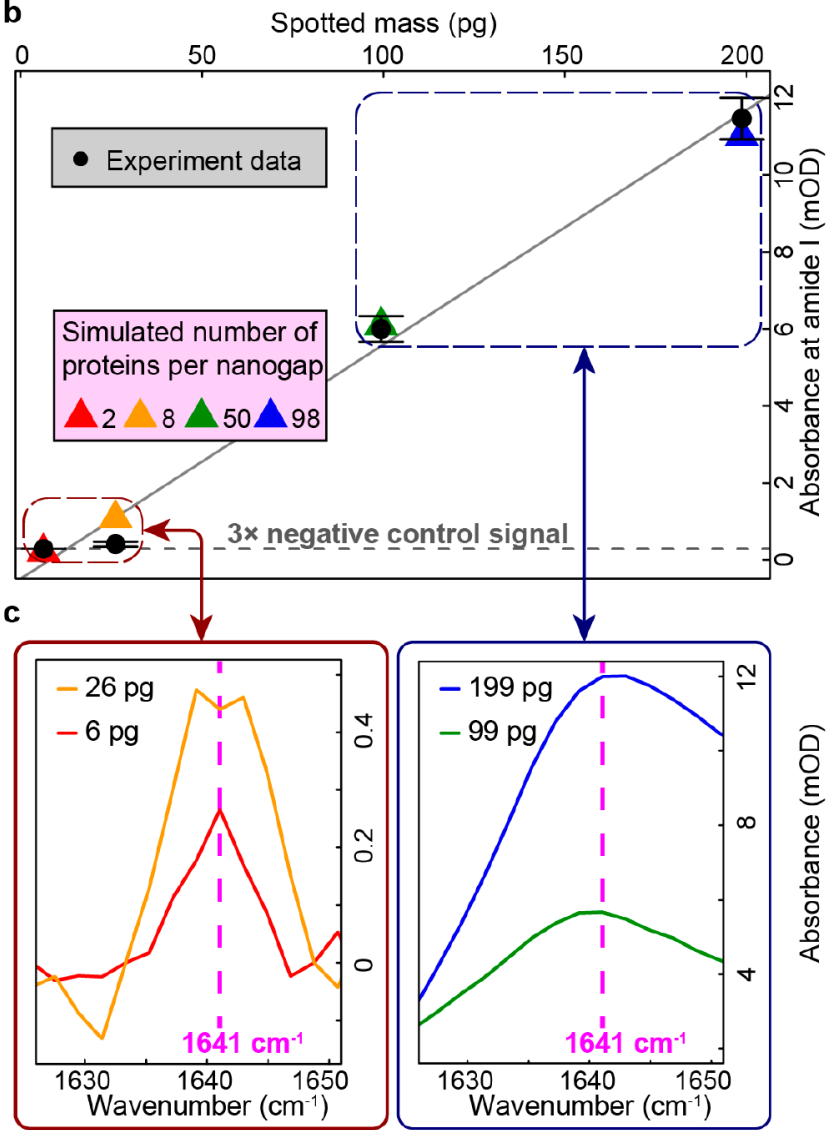

Figure 3. (a) Schematics depicting the experimental procedure for the preparation of chips with bound streptavidin proteins (not to scale) with the use of a microspotting dispenser. (b) Plot in which the black data points correspond to the experimentally measured absorbance at the amide I peak for different amounts of spotted protein mass. The colored triangular data points correspond to absorbance at the amide I peak obtained via simulations with different numbers of proteins per nanogap. (c) Exemplary absorbance curves which showcase the amide I peak. (d) 3D model of the simulation strategy followed to obtain the triangular data points shown in the plot of panel $\mathrm{b}$. The streptavidin proteins were homogeneously distributed in the nanogaps and modeled as $4 \times 5 \times 5 \mathrm{~nm}^{3}$ blocks with optical constants extracted from infrared reflection absorption spectroscopy (IRRAS) measurements (Figure S2b).

correspond to the periodicities for which the grating orders $\lambda_{0,-1}^{\mathrm{TE}}$ and $\lambda_{0, \pm 1}^{\mathrm{TM}}$, respectively, transition from the high frequency to the low frequency side of the antenna's localized resonance at $1600 \mathrm{~cm}^{-1}$. Therefore, to maximize both far-field and nearfield responses, we need to place the grating orders with the lowest frequencies directly at the high frequency side of the antenna far-field resonance in order to ensure that the antennas are in an evanescent regime, that is, their interacting electric field components add in phase to reduce the amount of energy that can escape the array as electromagnetic radiation. ${ }^{32}$

For increasing $y$-periodicity from 2 to $3.5 \mu \mathrm{m}$, the position of the lowest frequency grating order, that is, $\lambda_{0,-1}^{\mathrm{TE}}$, approaches the resonance from the high frequency side (Figure 2e), until it reaches $1711 \mathrm{~cm}^{-1}$, as can be seen as a kink in the reflectance curve in Figure $2 b$ (indicated by the vertical dashed line). The array is thus in a purely evanescent regime, as opposed to the case when $P_{y}=4 \mu \mathrm{m}$, for which the position of $\lambda_{0,-1}^{\mathrm{TE}}$ moves to the low frequency side of the far-field resonance (Figure 2e), thereby dampening the resonance in the TE channel and consequently decreasing the sensor's overall response (Figure $2 \mathrm{~d}$ ). For $P_{y}=4.5 \mu \mathrm{m}$, the position of $\lambda_{0,-1}^{\mathrm{TE}}$ is further in the radiative regime area, but now we also observe $\lambda_{0, \pm 1}^{\mathrm{TM}}$ at 1644 $\mathrm{cm}^{-1}$, that is, positioned at the high frequency side of the antenna resonance (Figure 2e). This yields an overall high performance of the sensor, however, not as high as for $P_{y}=3.5$ $\mu \mathrm{m}$. For $P_{y}=5 \mu \mathrm{m}$, the array is in a highly radiative regime which explains the drastically weakened sensor performance observed for the sensors featuring this $y$-periodicity value.

Within the explored parameter space, the sensor $\{G=20$ $\left.\mathrm{nm}, P_{y}=3.5 \mu \mathrm{m}\right\}$ yields the best performance for protein measurements. Consequently, we choose antenna parameters in the direct vicinity of this combination to obtain a highperformance sensor compatible with practical considerations regarding nanofabrication reproducibility and the size of the biomacromolecules. In particular, a gap size of $32 \mathrm{~nm}$ is chosen to accommodate a wider range of biomolecules with dimensions above $20 \mathrm{~nm}$, such as antibodies sandwiching an antigen (Figure $2 \mathrm{a}$ ), thus making our design broadly applicable to the study of proteins. Another parameter that influences the performance of antenna-based sensors is the decay characteristics of the electromagnetic near-fields around the hotspots. Through full-wave simulations of the electromagnetic fields, we have estimated the decay length as $125 \mathrm{~nm}$ for a $\{G=32 \mathrm{~nm}$, $\left.P_{y}=3204 \mathrm{~nm}\right\}$-GONG sensor (Figure S4b). This decay range makes the sensors effective for probing a large range of analyte molecules and changes in the environment.

In the following biomolecular studies in dry conditions, $\{G=$ $\left.32 \mathrm{~nm}, P_{y}=3204 \mathrm{~nm}\right\}$-GONG arrays with a size of $200 \times 200$ 


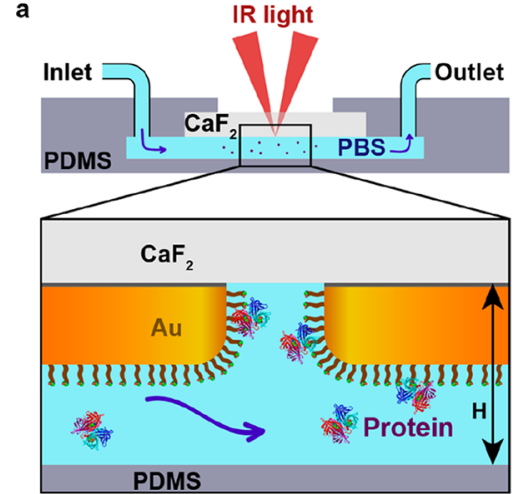

Microfluidics-integrated plasmonic chip

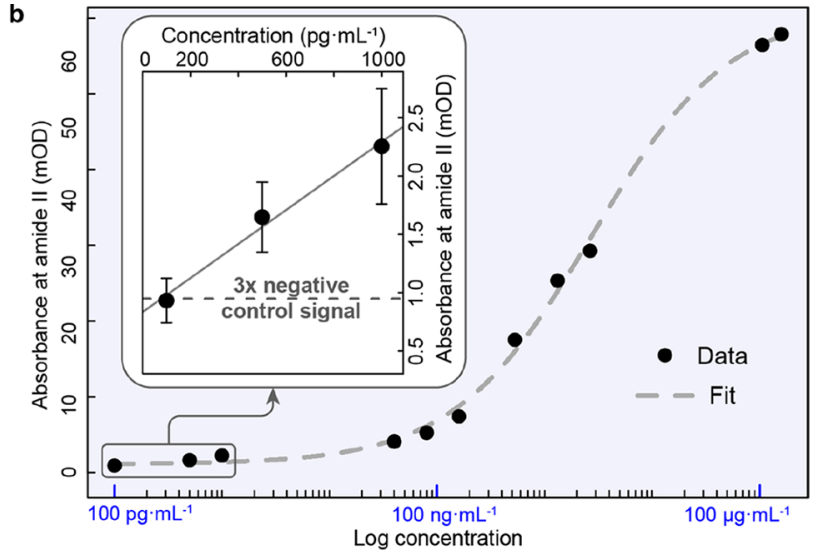

Figure 4. (a) Schematic of the in-solution experimental setup with the fluidic inlet and outlet as well as the optical path (not to scale). The flow channel height $H$ of the polydimethylsiloxane (PDMS) device is $30 \mu \mathrm{m}$. The zoomed schematic displays functionalized gold antennas displaying biotin groups and the captured proteins from the flowing solution. (b) Plot of absorbance at the amide II peak for different concentrations of streptavidin. A linear fit is displayed in the inset and a fit using eq 3 is displayed in the logarithmic plot.

$\mu \mathrm{m}^{2}$ are used for absorbance measurements using Fouriertransform infrared (FTIR) spectroscopy. To assess the performance of this optimized design for the detection of protein submonolayers, we perform experiments in which minute quantities of proteins are bound onto the antennas. Specifically, a piezoelectric microdispenser is used to spot droplets of controlled volume containing precise quantities of streptavidin in phosphate buffered saline (PBS) onto the antenna arrays (Figure 3a). The dispenser is equipped with a side camera to accurately measure the droplet size to be spotted on a specific region and by using this volume and the number of droplets being spotted, the total mass deposited on each sensor is controlled. Prior to spotting, the antenna surfaces are functionalized using biotinylated thiols. This allows us to probe the limit of amide I absorbance detection for specifically bound proteins on GONG arrays tuned to have their near-field resonance peaks at the amide I absorbance peak (Figure S4a).

Figure $3 \mathrm{~b}$ displays absorbance at the amide I peak against the spotted protein quantity. For spotted masses of 99 and 199 pg, the observed amide I absorbance is very strong compared to the noise level, but for spotted masses of 6 and $26 \mathrm{pg}$, we only observe the amide I absorbance peak at $1641 \mathrm{~cm}^{-1}$, just above three times the negative control signal level (Figure 3c). By using a surface-functionalization protocol, we ensure that our measured signals originate from specifically bound proteins. It is important to note that the measured surface masses are lower than the spotted mass quantities due to the thorough rinsing steps after incubation of the protein solutions. Thus, these values are conservative estimates and the measured signals should be generated from even lower quantities of analytes. Next, we correlate our measured signals with the corresponding number of proteins per nanogap using numerical simulations. We model the streptavidin proteins as blocks of $4 \times 5 \times 5 \mathrm{~nm}^{3}$, in agreement with reported streptavidin volumes ${ }^{40,41}$ and use optical constants extracted from IRRAS measurements (Figure S2b). In order to not overestimate the sensitivity of our sensors, we introduce a 1 $\mathrm{nm}$ thick layer with refractive index of 1.4 in between the antenna surface and the protein blocks to model the biotinylated thiol layer. Furthermore, we do not locate the blocks at the positions of the highest field enhancement, that is, the rounded corners and antenna/substrate intersections, but rather homogeneously space them on the gap surface (Figure $3 \mathrm{~d}$ ). Given that our antennas have a gap cross section of $100 \times$ $100 \mathrm{~nm}^{2}$, a maximum of approximately 800 proteins can be bound per nanogap surface area. We find that the numerical simulations for this case match the experimentally measured maximal signals very well, and similar results are obtained in the case of complete antenna coverage with a streptavidin layer (Figure S4c). This confirms that the simulations are in line with our measurements and that the signal predominantly originates from proteins within the gaps. From simulations with less than 800 proteins per nanogap, we can infer that measurements with spotted quantities of 199, 99, 26, and $6 \mathrm{pg}$ correspond to approximately $128,50,8$, and 2 proteins per nanogap.

Protein studies are usually conducted in solutions in order to maintain their native conformational state and biological function, therefore it is also crucial to establish the concentrations required for protein detection and secondary structure analysis in aqueous media. To perform in-solution absorbance measurements, we use $\left\{G=32 \mathrm{~nm}, P_{y}=3264\right.$ $\mathrm{nm}\}$-GONG arrays with a size of $200 \times 200 \mu \mathrm{m}^{2}$

Since our $\mathrm{CaF}_{2}$ substrate is highly transparent in the mid-IR range, we can illuminate our chips from the backside and integrate them within custom-made microfluidic devices, as shown in Figure 4a. By injecting streptavidin at increasing concentrations, we can establish the curve shown in Figure 4b. We chose this additive injection method due to the strong streptavidin-biotin interaction, which prohibits surface regeneration. The obtained S-shaped curve covers a large concentration range from $100 \mathrm{pg} \cdot \mathrm{mL}^{-1}$ to $100 \mu \mathrm{g} \cdot \mathrm{mL}^{-1}$. We fit the data using a Hill equation-derived fit, which empirically describes the correlation between the absorbance response $A$ of the biosensor and the concentration $C$ of the analytes in aqueous solution:

$$
A(C)=A_{\min }+\frac{A_{\max }-A_{\min }}{1+10^{n \cdot\left(C_{0.5}-C\right)}}
$$

Here, $A_{\min }$ and $A_{\max }$ correspond to the minimum and maximum absorbance signals, $C_{0.5}$ represents the concentration, which yields $\frac{A_{\max }}{2}, n$ is an exponent that is related to binding cooperativity, similarly to the Hill coefficient. ${ }^{42,43}$ In our case, we have $n<1$ in accordance with the expected 
negative cooperative binding behavior. The inset of Figure $4 \mathrm{~b}$ shows a zoomed linear plot for the lowest concentrations ranging from $100 \mathrm{pg} \cdot \mathrm{mL}^{-1}$ to $1 \mathrm{ng} \cdot \mathrm{mL}^{-1}$. The absorbance signal from an injection of $100 \mathrm{pg} \cdot \mathrm{mL}^{-1}$ streptavidin corresponds to approximately three times the absorbance signal from the injection of analyte-devoid buffer (negative control) and, thus, we can infer that our limit of detection is around $100 \mathrm{pg} \cdot \mathrm{mL}^{-1}$.

Using the same experimental data set, we can also estimate the concentration limit for secondary structure analysis. Information regarding the secondary structure of proteins is of great interest for unravelling complex biological processes ${ }^{44}$ such as protein misfolding, which play a major role in diseases including Alzheimer's and Parkinson's disease. ${ }^{45}$ Conventional IR spectroscopy of proteins in aqueous solution typically requires concentrations $>10 \mathrm{mg} \cdot \mathrm{mL}^{-1}$, which can be very challenging for the study of aggregation-prone proteins and peptides as well as rare samples. ${ }^{\text {It }}$ is thus of great interest to find new methods that can reduce the necessary concentrations for IR spectroscopy of proteins in aqueous solution. ${ }^{46}$ In fact, it has recently been shown in a pioneering work that the secondary structure of the protein $\alpha$-synuclein, which is involved in Parkinson's disease, can be analyzed in aqueous solution and in real-time using nanorod antennas. ${ }^{20}$ The amide I band of proteins mainly arises from the $\mathrm{C}=\mathrm{O}$ stretching of its backbone. This backbone configuration will influence the observed amide I absorbance spectrum as a consequence of transition dipole coupling between the different amide group oscillators and the particular hydrogen bonding patterns. Consequently, amide I absorbance spectra result from the superposition of sub-bands associated with different secondary structures present in a particular protein.

The secondary structure content of tetrameric streptavidin extracted from the current Uniprot database is $47.5 \% \beta$ strands, $22.4 \%$ disorder, $11.5 \%$ helices, and $18.5 \%$ loops and turns. The deconvolution of the absorbance spectrum obtained for a submonolayer of streptavidin in aqueous solution (Figure $5 \mathrm{a}$ ), which was obtained upon injection of $2.65 \mu \mathrm{g} \cdot \mathrm{mL}^{-1}$, yields a secondary structure content which agrees well with the database values, that is, we obtain $53.5 \% \beta$-strands, $16.9 \%$ disorder, $11.3 \%$ helices, and $18.3 \%$ loops and turns. Noticeably, we observe two bands for the helices centered at 1650 and $1666 \mathrm{~cm}^{-1}$, which are known to correspond to regular $\alpha$ helices and $33_{10}$-helices, respectively (Table 1 ). Furthermore, since in the literature the spectral position of turns tends to be reported at slightly higher wavenumbers than the spectral position of loops, we refine our analysis by associating the band at $1674 \mathrm{~cm}^{-1}$ with loops and the band at $1679 \mathrm{~cm}^{-1}$ with turns. This yields contents of $6.6 \%, 4.7 \%, 11.1 \%$, and $7.2 \%$ for $33^{-}$ helices, $\alpha$-helices, loops, and turns, respectively. These values are in good agreement with the average values calculated from Meskers et al.: ${ }^{47} 55.5 \% \beta$-strands, $19.4 \%$ disorder, $5.8 \% 3_{10^{-}}$ helices, $2.5 \% \alpha$-helices, $12.3 \%$ loops, and $4.5 \%$ turns.

By calculating the normalized root-mean-square error (NRMSE) for the spectra obtained for lower concentrations (Figure $5 b$ ) with respect to our scaled fit (Figure 5a), we see that we can retrieve the secondary structure content with high accuracy down to $530 \mathrm{ng} \cdot \mathrm{mL}^{-1}$, where the NMRSE remains below $1 \%$. For $160 \mathrm{ng} \cdot \mathrm{mL}^{-1}$, the NMRSE falls in the range 1$7.5 \%$, which still allows for secondary structure analysis but with reduced accuracy. For $80 \mathrm{ng} \cdot \mathrm{mL}^{-1}$ the NMRSE is in the range $7.5-15 \%$, in which it is no longer possible to reliably quantify the complete secondary structure content. Nonethe-
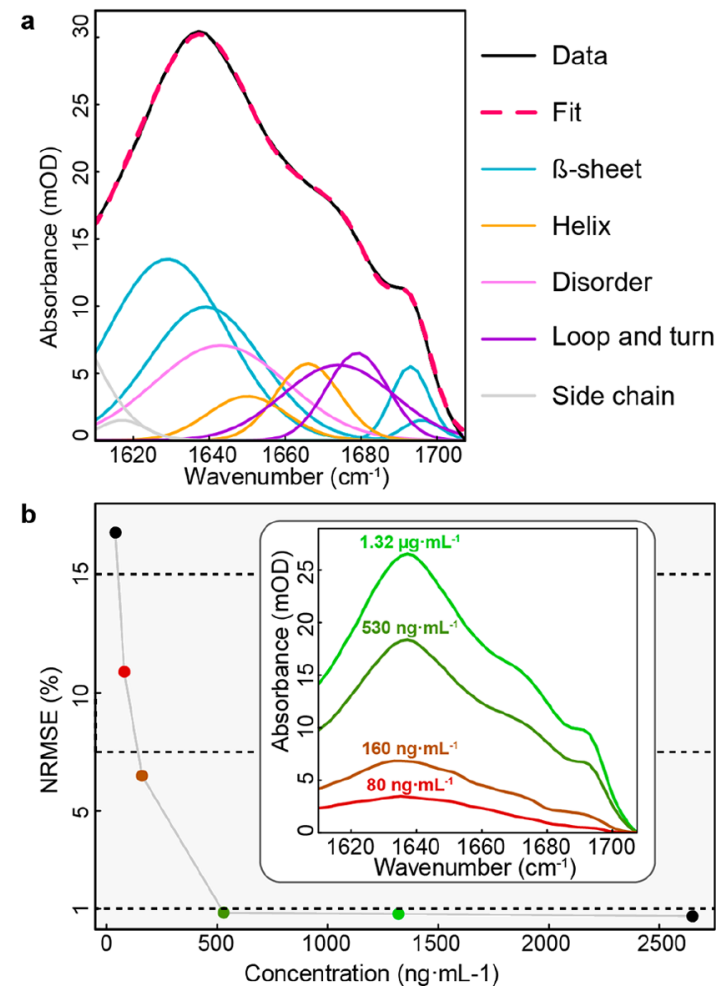

Figure 5. (a) Deconvoluted absorbance spectrum of a submonolayer of streptavidin in aqueous solution at a concentration of $2.65 \mu \mathrm{g} \cdot \mathrm{mL}^{-1}$ for quantitative identification of different secondary structure motifs, which are listed in the legend on the side. The pink dotted curve corresponds to the sum of Gaussian peaks which were used for fitting the experimental data (solid black curve). (b) The procedure shown in panel (a) is used to obtain the NRMSE for the experimental absorbance spectra at lower concentrations (colored dots) with respect to the (scaled) fit of the absorbance spectra shown with colored curves in the inset. The black dot with the lowest NRMSE corresponds to the data shown in panel (a).

Table 1. Secondary Structure Absorption Band Positions ${ }^{2,3}$

secondary structures
antiparallel $\beta$-strands
disorder
helices
loops and turns

loops and turn

less, it is still possible to retrieve the amide I peak position, which can be used to draw qualitative conclusions on the dominant secondary structure motif (antiparallel $\beta$-sheet in our case). Such information can provide important insights into biological processes, for example, as a means to diagnose prodromal Alzheimer's disease.

Even though our GONG design provides high sensitivities down to $\mathrm{ng} \cdot \mathrm{mL}^{-1}$ and $\mathrm{pg} \cdot \mathrm{mL}^{-1}$ levels, its performance could still be boosted if an increase in structural complexity is acceptable. For instance, it has been shown that the access of the enhanced near-fields to the target analyte can be increased by placing the plasmonic antennas on pedestals. ${ }^{49,50}$ Furthermore, in situations where a more spatially homogeneous near-field enhancement distribution is desired, other plasmonic designs such as ones based on annular gaps could be implemented. $^{28}$ 
In conclusion, we have introduced a framework for designing optimized nanogap arrays as well as assessing their performance for SEIRAS of minute quantities of biomolecules. We have used the optimized arrays for protein experiments and have shown that concentrations as low as $100 \mathrm{pg} \cdot \mathrm{mL}^{-1}$ can be detected in aqueous solution using protein-specific absorbance signals and that the secondary structure can be accurately retrieved at concentrations on the order of $500 \mathrm{ng} \cdot \mathrm{mL}^{-1}$. Our results emphasize the potentials of SEIRAS and nanoplasmonics for protein studies, as traditional IR spectroscopy would require orders of magnitude higher concentrations. In addition to protein analysis, GONG arrays can be adapted to the study of other classes of biomolecular systems such as lipid vesicles and exosomes, which currently attract significant interest due to their relevance to health and disease (Supporting Information, section 8). In this context, it has recently been shown that nanophotonic platforms based on metallic rods can resolve complex interaction processes in vesicular systems, such as the toxin-induced release of neurotransmitter molecules from synaptic vesicle mimics. ${ }^{18}$ The versatility of nanorod antennas for the study of a broad range of biological samples combined with the optimization principles presented in this work opens up exciting new applications in fields such as diagnostics and pharmacology.

\section{ASSOCIATED CONTENT}

\section{S Supporting Information}

The Supporting Information is available free of charge on the ACS Publications website at DOI: 10.1021/acsphotonics.8b00847.

Includes details on fabrication, numerical simulations, experimental measurements, and data analysis (PDF).

\section{AUTHOR INFORMATION}

\section{Corresponding Author}

*E-mail: hatice.altug@epfl.ch.

\section{ORCID}

Aurelian John-Herpin: 0000-0002-3554-1574

Andreas Tittl: 0000-0003-3191-7164

Hatice Altug: 0000-0001-5522-1343

\section{Notes}

The authors declare no competing financial interest.

\section{ACKNOWLEDGMENTS}

The streptavidin tetramer schematics displayed in Figure $1 b$, Figure 4a, and the Table of Contents artwork are adapted with permission from the work of the author Oxford grad on Wikimedia Commons under the Creative Commons Attribution license: CC BY-SA 3.0. The authors thank Dordaneh Etezadi for guidance regarding fabrication and functionalization of the plasmonic chips, Alexander Belushkin for help regarding fabrication and integration of the fluidic devices as well as Yashashwa Pandey for help with nanofabrication. The research leading to these results has received funding from the European Research Council (ERC) under Grant Agreement No. 682167 VIBRANT-BIO, the European Union Horizon 2020 Framework Programme for Research and Innovation under Grant Agreement Nos. 665667 (call 2015), FETOPEN737071 ULTRACHIRAL, and 777714 NOCTURNO. We also acknowledge École Polytechnique Fédérale de Lausanne
(EPFL) and Center of MicroNanoTechnology (CMI) for micro/nanofabrication.

\section{REFERENCES}

(1) Socrates, G. Infrared and Raman Characteristic Group Frequencies: Tables and Charts; John Wiley \& Sons, 2001.

(2) Barth, A. Infrared Spectroscopy of Proteins. Biochim. Biophys. Acta, Bioenerg. 2007, 1767 (9), 1073-1101.

(3) Yang, H.; Yang, S.; Kong, J.; Dong, A.; Yu, S. Obtaining Information about Protein Secondary Structures in Aqueous Solution Using Fourier Transform IR Spectroscopy. Nat. Protoc. 2015, 10 (3), 382-396.

(4) Cai, X.; Dass, C. Conformational Analysis of Proteins and Peptides. Curr. Org. Chem. 2003, 7 (18), 1841-1854.

(5) Wu, L.; Jiang, X. Infrared Spectroscopy for Studying Plasma Membranes. Membrane Biophysics; Springer: Singapore, 2018; pp 319-354.

(6) Baldassarre, M.; Barth, A. Pushing the Detection Limit of Infrared Spectroscopy for Structural Analysis of Dilute Protein Samples. Analyst 2014, 139 (21), 5393-5399.

(7) Neubrech, F.; Huck, C.; Weber, K.; Pucci, A.; Giessen, H. Surface-Enhanced Infrared Spectroscopy Using Resonant Nanoantennas. Chem. Rev. 2017, 117 (7), 5110-5145.

(8) Tabatabaei, M.; McRae, D.; Lagugné-Labarthet, F. Recent Advances of Plasmon-Enhanced Spectroscopy at Bio-Interfaces. Frontiers of Plasmon Enhanced Spectroscopy; American Chemical Society, 2016; Vol. 2, pp 183-207.

(9) Cerjan, B.; Yang, X.; Nordlander, P.; Halas, N. J. Asymmetric Aluminum Antennas for Self-Calibrating Surface-Enhanced Infrared Absorption Spectroscopy. ACS Photonics 2016, 3 (3), 354-360.

(10) Brown, L. V.; Yang, X.; Zhao, K.; Zheng, B. Y.; Nordlander, P.; Halas, N. J. Fan-Shaped Gold Nanoantennas above Reflective Substrates for Surface-Enhanced Infrared Absorption (SEIRA). Nano Lett. 2015, 15 (2), 1272-1280.

(11) Mayerhöfer, T. G.; Popp, J. Periodic Array-Based Substrates for Surface-Enhanced Infrared Spectroscopy. Nanophotonics 2018, 7 (1), $39-79$.

(12) Low, T.; Chaves, A.; Caldwell, J. D.; Kumar, A.; Fang, N. X.; Avouris, P.; Heinz, T. F.; Guinea, F.; Martin-Moreno, L.; Koppens, F. Polaritons in Layered Two-Dimensional Materials. Nat. Mater. 2017, 16 (2), 182-194.

(13) Yang, X.; Sun, Z.; Low, T.; Hu, H.; Guo, X.; García de Abajo, F. J.; Avouris, P.; Dai, Q. Nanomaterial-Based Plasmon-Enhanced Infrared Spectroscopy. Adv. Mater. 2018, 30 (20), 1704896.

(14) Wu, C.; Arju, N.; Kelp, G.; Fan, J. A.; Dominguez, J.; Gonzales, E.; Tutuc, E.; Brener, I.; Shvets, G. Spectrally Selective Chiral Silicon Metasurfaces Based on Infrared Fano Resonances. Nat. Commun. 2014, 5, 3892.

(15) Tittl, A.; Leitis, A.; Liu, M.; Yesilkoy, F.; Choi, D. Y.; Neshev, D. N.; Kivshar, Y. S.; Altug, H. Imaging-Based Molecular Barcoding with Pixelated Dielectric Metasurfaces. Science (Washington, DC, U. S.) 2018, 360 (6393), 1105-1109.

(16) Unser, S.; Bruzas, I.; He, J.; Sagle, L. Localized Surface Plasmon Resonance Biosensing: Current Challenges and Approaches. Sensors 2015, 15 (7), 15684-15716.

(17) Wallace, G. Q.; Foy, H. C.; Rosendahl, S. M.; LagugnéLabarthet, F. Dendritic Plasmonics for Mid-Infrared Spectroscopy. J. Phys. Chem. C 2017, 121 (17), 9497-9507.

(18) Rodrigo, D.; Tittl, A.; Ait-Bouziad, N.; John-Herpin, A.; Limaj, O.; Kelly, C.; Yoo, D.; Wittenberg, N. J.; Oh, S.-H.; Lashuel, H. A.; et al. Resolving Molecule-Specific Information in Dynamic Lipid Membrane Processes with Multi-Resonant Infrared Metasurfaces. Nat. Commun. 2018, 9 (1), 2160.

(19) Le, T. H. H.; Tanaka, T. Plasmonics-Nanofluidics Hydrid Metamaterial: An Ultrasensitive Platform for Infrared Absorption Spectroscopy and Quantitative Measurement of Molecules. ACS Nano 2017, 11 (10), 9780-9788.

(20) Etezadi, D.; Warner, J. B.; Lashuel, H. A.; Altug, H. Real-Time in-Situ Secondary Structure Analysis of Protein Monolayer with Mid- 
Infrared Plasmonic Nanoantennas. ACS Sensors 2018, 3 (6), 11091117.

(21) Morichika, I.; Kusa, F.; Takegami, A.; Sakurai, A.; Ashihara, S. Antenna-Enhanced Nonlinear Infrared Spectroscopy in Reflection Geometry. J. Phys. Chem. C 2017, 121 (21), 11643-11649.

(22) Adato, R.; Altug, H. In-Situ Ultra-Sensitive Infrared Absorption Spectroscopy of Biomolecule Interactions in Real Time with Plasmonic Nanoantennas. Nat. Commun. 2013, 4, 2154.

(23) Limaj, O.; Etezadi, D.; Wittenberg, N. J.; Rodrigo, D.; Yoo, D.; $\mathrm{Oh}, \mathrm{S}$.; Altug, H. Infrared Plasmonic Biosensor for Real-Time and Label-Free Monitoring of Lipid Membranes. Nano Lett. 2016, 16, 1502.

(24) Vogt, J.; Huck, C.; Neubrech, F.; Pucci, A. Plasmonic Light Scattering and Infrared Vibrational Signal Enhancement. Frontiers of Plasmon Enhanced Spectroscopy; American Chemical Society, 2016; Vol. 2, pp 1-19.

(25) Ciracì, C.; Hill, R. T.; Mock, J. J.; Urzhumov, Y.; FernándezDomínguez, A. I.; Maier, S. A.; Pendry, J. B.; Chilkoti, A.; Smith, D. R. Probing the Ultimate Limits of Plasmonic Enhancement. Science (Washington, DC, U. S.) 2012, 337 (6098), 1072-1074.

(26) Ji, D.; Cheney, A.; Zhang, N.; Song, H.; Gao, J.; Zeng, X.; Hu, H.; Jiang, S.; Yu, Z.; Gan, Q. Efficient Mid-Infrared Light Confinement within Sub-5-Nm Gaps for Extreme Field Enhancement. Adv. Adv. Opt. Mater. 2017, 5 (17), na.

(27) Huck, C.; Neubrech, F.; Vogt, J.; Toma, A.; Gerbert, D.; Katzmann, J.; Härtling, T.; Pucci, A. Surface-Enhanced Infrared Spectroscopy Using Nanometer-Sized Gaps. ACS Nano 2014, 8 (5), $4908-4914$.

(28) Yoo, D.; Mohr, D. A.; Vidal-Codina, F.; John-Herpin, A.; Jo, M.; Kim, S.; Matson, J.; Caldwell, J. D.; Jeon, H.; Nguyen, N. C.; et al. High-Contrast Infrared Absorption Spectroscopy via Mass-Produced Coaxial Zero-Mode Resonators with Sub-10 Nm Gaps. Nano Lett. 2018, 18 (3), 1930-1936.

(29) Chen, X.; Ciracì, C.; Smith, D. R.; Oh, S. H. NanogapEnhanced Infrared Spectroscopy with Template-Stripped Wafer-Scale Arrays of Buried Plasmonic Cavities. Nano Lett. 2015, 15 (1), 107113.

(30) Hoffmann, J. M.; Janssen, H.; Chigrin, D. N.; Taubner, T. Enhanced Infrared Spectroscopy Using Small-Gap Antennas Prepared with Two-Step Evaporation Nanosphere Lithography. Opt. Express 2014, 22 (12), 14425-14432.

(31) Dong, L.; Yang, X.; Zhang, C.; Cerjan, B.; Zhou, L.; Tseng, M. L.; Zhang, Y.; Alabastri, A.; Nordlander, P.; Halas, N. J. Nanogapped $\mathrm{Au}$ Antennas for Ultrasensitive Surface-Enhanced Infrared Absorption Spectroscopy. Nano Lett. 2017, 17 (9), 5768-5774.

(32) Adato, R.; Yanik, A. A.; Amsden, J. J.; Kaplan, D. L.; Omenetto, F. G.; Hong, M. K.; Erramilli, S.; Altug, H. Ultra-Sensitive Vibrational Spectroscopy of Protein Monolayers with Plasmonic Nanoantenna Arrays. Proc. Natl. Acad. Sci. U. S. A. 2009, 106 (46), 19227-19232.

(33) Bagheri, S.; Weber, K.; Gissibl, T.; Weiss, T.; Neubrech, F.; Giessen, H. Fabrication of Square-Centimeter Plasmonic Nanoantenna Arrays by Femtosecond Direct Laser Writing Lithography/ Effects of Collective Excitations on SEIRA Enhancement. ACS Photonics 2015, 2 (6), 779-786.

(34) Yang, Z.; Kollman, J. M.; Pandi, L.; Doolittle, R. F. Crystal Structure of Native Chicken Fibrinogen at $2.7 \AA$ Resolution. Biochemistry 2001, 40 (42), 12515-12523.

(35) Diebold-Durand, M. L.; Lee, H.; Avila, L. B. R.; Noh, H.; Shin, H. C.; Im, H.; Bock, F. P.; Bürmann, F.; Durand, A.; Basfeld, A.; et al. Structure of Full-Length SMC and Rearrangements Required for Chromosome Organization. Mol. Cell 2017, 67 (2), 334-347.

(36) Chuang, C. K.; Rockel, B.; Seyit, G.; Walian, P. J.; Schönegge, A. M.; Peters, J.; Zwart, P. H.; Baumeister, W.; Jap, B. K. Hybrid Molecular Structure of the Giant Protease Tripeptidyl Peptidase II. Nat. Struct. Mol. Biol. 2010, 17 (8), 990-996.

(37) Vestergaard, B.; Groenning, M.; Roessle, M.; Kastrup, J. S.; Van De Weert, M.; Flink, J. M.; Frokjaer, S.; Gajhede, M.; Svergun, D. I. A Helical Structural Nucleus Is the Primary Elongating Unit of Insulin Amyloid Fibrils. PLoS Biol. 2007, 5 (5), e134.
(38) Liberman, V.; Adato, R.; Mertiri, A.; Yanik, A. A.; Chen, K.; Jeys, T. H.; Erramilli, S.; Altug, H. Angle-and Polarization-Dependent Collective Excitation of Plasmonic Nanoarrays for Surface Enhanced Infrared Spectroscopy. Opt. Express 2011, 19 (12), 11202-11212.

(39) Maß, T. W. W.; Taubner, T. Incident Angle-Tuning of Infrared Antenna Array Resonances for Molecular Sensing. ACS Photonics 2015, 2 (10), 1498-1504.

(40) Helm, C. A.; Knoll, W.; Israelachvili, J. N. Measurement of Ligand-Receptor Interactions. Proc. Natl. Acad. Sci. U. S. A. 1991, 88 (18), 8169-8173.

(41) Reiter, R.; Motschmann, H.; Knoll, W. Ellipsometric Characterization of Streptavidin Binding to Biotin-Functionalized Lipid Monolayers at the Water/Air Interface. Langmuir 1993, 9 (9), 2430-2435.

(42) Keizer, H. M.; Dorvel, B. R.; Andersson, M.; Fine, D.; Price, R. B.; Long, J. R.; Dodabalapur, A.; Köper, I.; Knoll, W.; Anderson, P. A.; et al. Functional Ion Channels in Tethered Bilayer Membranes Implications for Biosensors. ChemBioChem 2007, 8 (11), 1246-1250.

(43) Wittenberg, N. J.; Johnson, T. W.; Oh, S. H. High-Density Arrays of Submicron Spherical Supported Lipid Bilayers. Anal. Chem. 2012, 84 (19), 8207-8213.

(44) Jackman, J. A.; Ferhan, A. R.; Cho, N.-J. Nanoplasmonic Sensors for Biointerfacial Science. Chem. Soc. Rev. 2017, 46 (12), 3615-3660.

(45) Lansbury, P. T.; Lashuel, H. A. A Century-Old Debate on Protein Aggregation and Neurodegeneration Enters the Clinic. Nature 2006, 443 (7113), 774-779.

(46) Etezadi, D.; Warner, J. B.; Ruggeri, F. S.; Dietler, G.; Lashuel, H. A.; Altug, H. Nanoplasmonic Mid-Infrared Biosensor for in Vitro Protein Secondary Structure Detection. Light: Sci. Appl. 2017, 6 (8), e17029.

(47) Meskers, S.; Ruysschaert, J. M.; Goormaghtigh, E. HydrogenDeuterium Exchange of Streptavidin and Its Complex with Biotin Studied by 2D-Attenuated Total Reflection Fourier Transform Infrared Spectroscopy. J. Am. Chem. Soc. 1999, 121 (22), 5115-5122.

(48) Nabers, A.; Ollesch, J.; Schartner, J.; Kötting, C.; Genius, J.; Hafermann, H.; Klafki, H.; Gerwert, K.; Wiltfang, J. Amyloid- $\beta$ Secondary Structure Distribution in Cerebrospinal Fluid and Blood Measured by an Immuno-Infrared-Sensor: A Biomarker Candidate for Alzheimer's Disease. Anal. Chem. 2016, 88 (5), 2755-2762.

(49) Cetin, A. E.; Etezadi, D.; Altug, H. Accessible Nearfields by Nanoantennas on Nanopedestals for Ultrasensitive Vibrational Spectroscopy. Adv. Opt. Mater. 2014, 2 (9), 866-872.

(50) Huck, C.; Toma, A.; Neubrech, F.; Chirumamilla, M.; Vogt, J.; De Angelis, F.; Pucci, A. Gold Nanoantennas on a Pedestal for Plasmonic Enhancement in the Infrared. ACS Photonics 2015, 2 (4), 497-505. 